\title{
Partitioning a graph into minimum gap components
}

\author{
Maurizio Bruglieri ${ }^{1}$ \\ Dipartimento di Design, Politecnico di Milano, Milano, Italy
}

Roberto Cordone ${ }^{2}$

Dipartimento di Informatica, Università degli Studi di Milano, Milano, Italy

\begin{abstract}
We study the computational complexity and approximability for the problem of partitioning a vertex-weighted undirected graph into $p$ connected subgraphs with minimum gap between the largest and the smallest vertex weights.
\end{abstract}

Keywords: Graph partitioning, computational complexity, approximability

\section{Introduction}

Let $G=(V, E)$ be an undirected connected graph, $w_{v}$ an integer weight coefficient defined on each vertex $v \in V$, and $p \leq|V|$ a positive integer number. Given a vertex subset $U \subseteq V$, we denote by $m_{U}=\min _{u \in U} w_{u}$ and $M_{U}=\max _{u \in U} w_{u}$ the minimum and maximum weight in $U$, respectively, and by gap their difference $\gamma_{U}=M_{U}-m_{U}$. The Minimum Gap Graph Partitioning

1 Email: maurizio.bruglieri@polimi.it

2 Email: roberto.cordone@unimi.it 
Problem ( $M G G P P$ ) requires to partition $G$ into $p$ vertex-disjoint connected subgraphs $G_{r}=\left(V_{r}, E_{r}\right),(r=1, \ldots, p)$ with at least two vertices each. Its min-max and min-sum versions minimize, respectively, the maximum gap $f^{M M}$ and the sum of the gaps $f^{M S}$ over all subgraphs:

$$
f^{M M}=\max _{r=1, \ldots, p} \gamma_{V_{r}} \quad f^{M S}=\sum_{r=1}^{p} \gamma_{V_{r}}
$$

The $M G G P P$ can find applications in agriculture (divide a land into parcels with limited difference in height [3]), in the location of gate houses along rivers, and in social network analysis (identify connected clusters of members with homogeneous features). It falls in the large field of graph partitioning problems [1,2], but, as far as we know, objective functions related to the differences between vertex weights in each subgraph have never been considered before.

\section{Complexity}

Theorem 2.1 The MGGPP admits feasible solutions if and only if graph $G$ contains a matching of cardinality at least $p$.

Proof. Any maximum cardinality matching $M$ induces on graph $G$ a spanning forest of $|M|$ nondegenerate trees and $|V|-2|M|$ isolated vertices. Each isolated vertex $v$ has an incident edge $e_{v}$ which is adjacent to an edge in $M$. Adding $e_{v}$ to $M$ for each isolated vertex $v$, we obtain a spanning forest of exactly $|M|$ trees. If $|M|>p$, we consider the edges connecting different trees, and we add them to $M$, stopping as soon as we obtain exactly $p$ trees. This provides a feasible solution of the $M G G P P$. Vice versa, given a feasible solution, we can choose an edge from each subgraph (they all contain at least two vertices): these edges are nonadjacent, and yield a $p$-cardinality matching.

Let $W_{U}=\left\{z \in \mathbb{Z}: \exists v \in U\right.$ with $\left.w_{v}=z\right\}$ be the set of values assumed by $w$ on a subset of vertices $U \subseteq V$, and $\eta_{U}=\left|W_{U}\right|$ the number of such values.

Theorem 2.2 The MGGPP with the min-max objective function is strongly $\mathcal{N P}$-hard even if $p=2$ and $\eta_{V}=3$.

Proof. The decision version of the problem, obviously in $\mathcal{N} \mathcal{P}$, amounts to verifying the existence of a solution such that the gap of all subgraphs is not larger than a given threshold. Given a generic instance of $S A T$, we build the following auxiliary graph. We introduce for each literal $\left(x_{i}\right.$ or $\left.\bar{x}_{i}\right)$ a vertex $\left(v_{i}\right.$ or $\left.\bar{v}_{i}\right)$ with $w_{v_{i}}=w_{\bar{v}_{i}}=2$, and for each clause $C_{j}$ a vertex $c_{j}$ with weight $w_{c_{j}}=1$; 


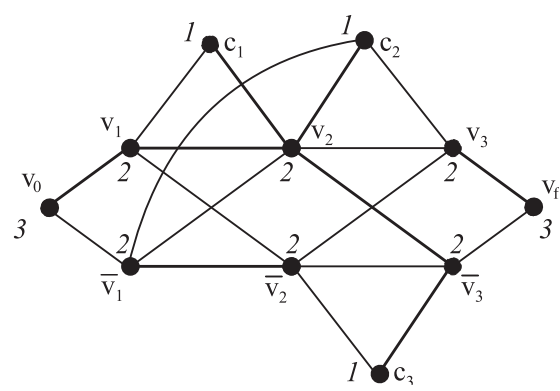

Fig. 1. Graph construction for the $\mathcal{N} \mathcal{P}$-hardness proof of the min-max $M G G P P$

finally, we introduce two dummy vertices $v_{0}$ and $v_{f}$ with weight $w_{0}=w_{f}=3$. Vertex $v_{0}$ is connected to $v_{1}$ and $\bar{v}_{1}$; vertex $v_{f}$ is connected to $v_{n}$ and $\bar{v}_{n}$; each vertex $v_{i}\left(\right.$ resp. $\left.\bar{v}_{i}\right)$ is connected to $v_{i+1}$ and $\bar{v}_{i+1}(i=1, \ldots, n-1)$ and to all the clause vertices $c_{j}$ such that literal $x_{i}$ (resp. $\bar{x}_{i}$ ) occurs in clause $C_{j}$. We are looking for $p=2$ connected subgraphs with gaps not larger than 1 . Figure 1 shows the graph corresponding to $\left(x_{1} \vee x_{2}\right) \wedge\left(\bar{x}_{1} \vee x_{2} \vee x_{3}\right) \wedge\left(\bar{x}_{2} \vee \bar{x}_{3}\right)$. If both subgraphs have gap $\leq 1, v_{0}$ and $v_{f}$ belong to the same subgraph, and this connects them through a path entirely made of vertices $v_{i}$ or $\bar{v}_{i}$. By construction, this path contains at least one of $v_{i}$ or $\bar{v}_{i}$ for each variable $x_{i}$. The other subgraph contains all the clause vertices $c_{j}$ and connects them through adjacent vertices $v_{i}$ or $\bar{v}_{i}$ which identify literals satisfying all clauses. Such a truth assignment is consistent because the subgraph includes at most one vertex for each variable $x_{i}$. Vice versa, any satisfying truth assignment identifies a partition of the graph into two subgraphs with gap $\leq 1$.

Theorem 2.3 The MGGPP with the min-sum objective function is strongly $\mathcal{N P}$-hard even if $\eta_{V}=2$.

Proof (Sketch). The proof is by reduction from 3-SAT.

\section{Approximability}

Theorem 3.1 The min-max MGGPP cannot be approximated for any constant $\alpha<2$ unless $\mathcal{P}=\mathcal{N} \mathcal{P}$.

Proof. Following Theorem 2.2, we can build an instance with optimum equal to 1 for any YES-instance of $S A T$ and one with optimum equal to 2 for any NO-instance. By contradiction, a hypothetical $\alpha$-approximated polynomial algorithm with $\alpha<2$, would find on the former instances solutions with a value $<2$ (by integrality, 1), and therefore solve $S A T$ in polynomial time.

Theorem 3.2 The MGGPP is 2-approximable for $p=2$. 
Proof. Let $V_{1}^{*}$ and $V_{2}^{*}$ be the unknown subsets of vertices of the optimal solution. The ranges of the weights in the two subgraphs, $\left[m_{V_{1}^{*}} ; M_{V_{1}^{*}}\right]$ and $\left[m_{V_{2}^{*}} ; M_{V_{2}^{*}}\right]$, are either separate or overlapping. In the former case, all the vertices in a subgraph have weights strictly smaller than those in the other. Then, the optimal solution can be found by exhaustively considering all pairs of intervals $\left[w_{\pi_{1}}, w_{\pi_{k}}\right]$ and $\left[w_{\pi_{k+1}}, w_{\pi_{\eta}}\right]\left(k=1, \ldots, \eta_{V}-1\right)$, and building the subgraphs induced on $G$ by the vertices whose weights fall in the two intervals. In the latter case, the two ranges overlap, and $f^{* M S}=\gamma_{V_{1}^{*}}+\gamma_{V_{2}^{*}} \geq \gamma_{V}$, which implies $f^{* M M}=\max \left(\gamma_{1}^{*}, \gamma_{2}^{*}\right) \geq \gamma_{V} / 2$. Generating any feasible solution with Theorem 2.1, we obtain $f^{M S} \leq 2 \gamma_{V} \leq 2 f^{* M S}$ and $f^{M M} \leq \gamma_{V} \leq 2 f^{* M M}$.

\section{Some special cases}

The MGGPP admits some polynomially solvable special cases.

Proposition 4.1 The min-max MGGPP is polynomially solvable if $\eta_{V}=2$.

Proof (Sketch). If there is a vertex whose weight is different from that of the adjacent vertices, the optimal solution is $\gamma_{V}$. Otherwise, we merge all the adjacent vertices of equal weight and consider the resulting vertex set $V^{\prime}$. If $\left|V^{\prime}\right|>p$, the optimum is $\gamma_{V}$; otherwise, a procedure similar to that of Theorem 2.1 provides an optimal solution with $p$ subgraphs of zero gap.

Proposition 4.2 The min-sum and min-max MGGPP are polynomially solvable on line graphs.

Proof (Sketch). The proof is based on the computation by dynamic programming of the minimum bottleneck path on a suitable graph.

We are currently investigating the complexity of other special cases and working on the design of exact and heuristic algorithms.

\section{References}

[1] D. A. Bader, H. Meyerhenke, P. Sanders, and D. Wagner, eds. Graph Partitioning and Graph Clustering, v. 588 of Contemporary Mathematics. AMS, 2013.

[2] C.-E. Bichot and P. Siarry, editors. Graph Partitioning. Wiley-ISTE, 2013.

[3] Li Xiao, Li Hongpeng, Niu Dongling, Wang Yan, and Liu Gang. Optimization of GNSS-controlled land leveling system and related experiments. Transactions of the Chinese Society of Agricultural Engineering, 31(3):48-55, 2015. 